\title{
A Multiple Indexes Quality Assessment for Fingerprint
}

\author{
Sheng Chang*, Qijun Huang, Hao Wang, Jin He \\ School of Physics and Technology, Wuhan University, Wuhan 430072, China \\ email: changsheng@whu.edu.cn
}

Keywords: Quality Estimation; Support Vector Machine; Wavelet Transform; Harris Corner

\begin{abstract}
The performance of fingerprint recognition relies heavily on the quality of fingerprint images. In this paper, a multiple indexes fingerprint quality assessment is proposed for poor fingerprints. This method fuses seven indexes from three kinds of typical fingerprint features (gray features, local features and global features) by a support vector machine classifier. Experiment results on FVC2004 database show that our proposed method can identify poor quality fingerprint accurately (94.3\% -98.7\% for different sub databases).
\end{abstract}

\section{Introduction}

Poor quality images can introduce spurious information or miss minutiae, and thus degrade the performance of automated fingerprint identification systems (AFISs). To deal with poor quality fingerprints, some attentions focused on the back-end of recognition, such as feature code and minutiae matching [1]. But if the poor quality fingerprints can be estimated first, the press on back-end will be released and the performance of AFISs can be improved.

About estimation index for fingerprint quality assessment, most studies limited their identification on just one kind of features. Gradient matrix characteristic of the orientation certainty localization (OCL) was used in [2]. In [3], principal component analysis (PCA) was employed by exploiting Harris corner strength (HCS) [4] as a weighted value. Energy concentration in frequency domain [5] well reflected the overall quality of a fingerprint, but it lacked the characteristics from local regions. In [6], wavelet based fingerprint image quality estimation was presented, but it is only applicable for the quality assessment of wavelet scalar quantization (WSQ) compressed images. There is still a bright potential to fuse them together.

On the other aspect, estimation method was explored by using single index analysis, multivariate linear weighted [7] and multi-index nonlinear fusion [8]. Artificial Neural Network (ANN) and Back Propagation Neural Network (BPNN) based nonlinear classifiers were developed [9], respectively. A two-step BPNN classifier based on optimal orientation certainty was proposed further [10]. But generally speaking, the NN approach is effective for high dimension and mass sample data, which is not very suitable for fingerprints.

Upon the above analysis, to develop the discrimination method of low quality fingerprints, a poor quality fingerprint assessment method is proposed in this paper. The estimation fuses seven indexes from three kinds of typical fingerprint features (gray features, local features and global features), and classifies fingerprints by another intelligent computing method, support vector machine (SVM). At the rest of the paper, the quality indexes are presented firstly, and then the SVM classification is given. Finally, the performance of our method is verified.

\section{Fingerprint quality indexes}

\section{Gray features}

The ratio value $Q_{\text {area }}$ derived by Eq. (1) identifies the proper effective area. $N_{\text {foreground }}$ is the block numbers of foreground, which is segmented based on variance, and $N_{\text {image }}$ is the total number of blocks (For example, an image of $256 \times 256$ pixels can be divided to $32 \times 32$ blocks). By the way, if 
$Q_{\text {area }}<s 1$, the effective area is too small to be usable for AFIS. Variable $s 1(0<s 1 \leq 1)$ depends on application requirements.

$$
Q_{\text {area }}=N_{\text {foreground }} / N_{\text {image }}
$$

The moisture of fingerprint is an important characteristic of gray features. The ridge lines of dry fingerprint usually contain many fracture places. The ridge-valley borders of wet fingerprint are not clear enough, and may be blur and dark. As a result, the moisture affects the reliability of fingerprint minutiae extraction. This characteristic can be represented by the mean gray $Q_{\text {mean }}$ and the gray variance $Q_{\mathrm{var}}$ in the foreground region. The dry fingerprint images have high gray mean and low gray variance, while the wet ones have relative low gray mean and high gray variance.

\section{Global features}

Our global features include quality indexes in frequency and wavelet domain. The fluctuation of ridge and valley, belonging to a narrow frequency band, results in an obvious annular structure in the frequency domain. The energy difference of this annular structure can be defined as a global index $Q_{f f t}$ in Eq. (2). In which, $C_{n s t}$ is a normalized constant and $E_{f f t}$ is the difference of spectrum value between the largest one and its two neighbors. $f(\theta, r)$ denotes the largest spectrum value along $r$ direction (our experienced value range of $r$ is from 25 to 50) for a given $\theta$. Besides, $f(\theta, r / 2)$ and $f(\theta, 1.5 \times r)$ are the largest spectrum values along $r / 2$ and $3 \times r / 2$, respectively.

$$
Q_{f f t}=\frac{1}{C_{n s t}} \times \frac{1}{360} \sum_{\theta=1}^{360} E_{f f t}, \quad E_{f f t}=2 \times f(\theta, r)-f(\theta, r / 2)-f(\theta, 3 \times r / 2)
$$

Besides the Fourier analysis, a texture analysis method based on wavelet transform (WT) is proposed. A fingerprint image is decomposed to $N$ layers by WT. For an image of $M \times M$ pixels, $D_{n}^{i} f$ denotes the high frequency coefficient of the $N$ 'th layer. Sub-band $s$ represents the component of horizontal $(s=1)$, vertical $(s=2)$ and diagonal $(s=3)$. Energy $E_{\text {wavelet }}$ is the sum of high frequency components of $N$ layers denoted. A fingerprint image is decomposed into three layers based on Haar wavelet in this paper. The global wavelet index $Q_{\text {wavelet }}$ is defined in Eq. (3) according to the energy of high frequency components. In which, $C_{w}$ is the normalized constant for a specific database.

$$
\mathrm{Q}_{\text {wavelet }}=\frac{1}{C_{w}} \times E_{\text {wavelet }}, \quad E_{\text {wavelet }}=\sum_{s=1}^{3} \sum_{n=1}^{N}\left|D_{n}^{s} f\right|^{2}
$$

\section{Local features}

To improve the quality estimation accuracy of poor fingerprints, OCL and HCS are employed as indexes of local features in this paper. The orientation and intensity of a pixel can be represented by its gray gradients ( $d x, d y$ ). In a $N_{T}$-points image block, the covariance matrix $C$ is given by Eq. (4), where $d x$ and $d y$ are the horizontal and vertical gradients (Sobel operator) of each pixel, respectively. The two eigenvalues of matrix $C$ are denoted by $\lambda_{\max }$ and $\lambda_{\min }$ in Eq. (5). In which, $\lambda_{\max }$ is the eigenvalue representing the direction having the largest curvature, while $\lambda_{\min }$ represents the direction of the least curvature. The OCL index of each block is defined as $Q_{o c l}$, and the normalized OCL index is $Q_{O C L}$.

$$
\begin{aligned}
& \mathrm{C}=\frac{1}{N_{T}}\left\{\left[\frac{d x}{d y}\right][d x d y]\right\}=\frac{1}{N_{T}}\left[\begin{array}{cc}
d x^{2} & d x d y \\
d x d y & d y^{2}
\end{array}\right]=\frac{1}{N_{T}}\left[\begin{array}{cc}
c_{x x} & c_{x y} \\
C_{x y} & c_{y y}
\end{array}\right] \\
& \lambda_{\text {max }}=\frac{c_{x x}+c_{y y}+\sqrt{\left(c_{x x}-c_{y y}\right)^{2}+4 c_{x y}{ }^{2}}}{2}, \quad \lambda_{\min }=\frac{c_{x x}+c_{y y}-\sqrt{\left(c_{x x}-c_{y y}\right)^{2}+4 c_{x y}{ }^{2}}}{2} \\
& Q_{O C L}=\frac{1}{N_{\text {foreground }}} \sum_{k=1}^{N_{\text {foreground }}} Q_{o c l}, \quad Q_{o c l}=1-\frac{\lambda_{\min }}{\lambda_{\max }}
\end{aligned}
$$

The quality index $\left(Q_{H C S}\right)$ represented by HCS of a fingerprint image is shown in Eq. (7), where $Q_{r}$ is the quality index of each block and is determined by Eq. (8). The gradients $\left(G_{x}, G_{y}\right)$ at pixel 
position $(i, j)$ are obtained using the complex Marr-Hildreth operator and $C_{r}$ is a normalized constant.

$$
\begin{aligned}
& Q_{H C S}=\frac{1}{N_{\text {foreground }}} \sum_{k=1}^{N_{\text {freground }}} Q_{r}, \quad Q_{r}=\frac{1}{C_{r}} \frac{I_{x}^{2} I_{y}^{2}-I_{x y}^{2}}{I_{x}^{2}+I_{y}^{2}} \\
& I_{x}^{2}=\sum_{i=1}^{W} \sum_{j=1}^{W} G_{x}^{2}(i, j), \quad I_{y}^{2}=\sum_{i=1}^{W} \sum_{j=1}^{W} G_{y}^{2}(i, j), \quad I_{x y}^{2}=\sum_{i=1}^{W} \sum_{j=1}^{W} G_{x}^{2}(i, j) G_{y}^{2}(i, j)
\end{aligned}
$$

\section{Classification based on SVM}

When the seven quality indexes are extracted, the quality of a fingerprint image is estimated by support vector machine. SVM classifier bases on a structural risk minimization theory. It provides linear separation in an augmented space by means of different kernels with excellent generalization capability. Besides, it has an advantage of much notable with a small training set and high data dimension. Each instance in the training set contains one target value (fingerprint quality level or score) and several attributes (extracted features). This method exploits the fusion of multi-indexes, which avoids the limitation of single index. Thus it is more applicable for different fingerprint databases. Three basic kernels (linear, polynomial and radial basis function (RBF)) are used to compare kernel's effect on estimation in this paper. The linear, polynomial and RBF kernels are determined by Eq. (9), Eq. (10) and Eq. (11), respectively. In which, $\gamma, r$ and $d$ are the kernel parameters.

$$
\begin{aligned}
& K_{\text {linear }}\left(x_{i}, x_{j}\right)=x_{i}^{T} x_{j} \\
& K_{\text {polynomial }}\left(x_{i}, x_{j}\right)=\left(\gamma x_{i}^{T} x_{j}+r\right)^{d}, \gamma>0 \\
& K_{R B F}\left(x_{i}, x_{j}\right)=\exp \left(-\gamma\left\|x_{i}-x_{j}\right\|^{2}\right), \gamma>0
\end{aligned}
$$

The extracted indexes (seven indexes in three features) denote the input vector of SVM for classifying. The quality of a fingerprint image is estimated by the C-support vector classification (C-SVC). The input vector is shown as Eq. (12).

$$
V=\left[Q_{\text {area }}, Q_{\text {mean }}, Q_{\text {var }}, Q_{\text {fft }}, Q_{\text {wavelet }}, Q_{O C L}, Q_{H C S}\right]
$$

\section{Experiment results and discussion}

To evaluate the accuracy and the efficiency of the proposed method, experiments of quality assessment are validated. FVC2004 is chosen as the fingerprint dataset. The fingerprints of sub databases DB1 and DB2 are captured by optical sensors and DB3 is collected by thermal devices. There are four, three and two classes in DB1, DB2 and DB3, respectively. Each class contains 200 images (100 images for training and the rest for predicting).

To evaluate the efficiency of the proposed quality estimation of fingerprint, DB2 is used in the experiment, which includes the quality of dry, wet and good. Different quality indexes are validated individually and jointly to demonstrate their contribution to the accuracy of quality estimation. The accuracy of different quality indexes for fingerprint quality estimation is illustrated in Fig. 1. It can be seen that multiple features can obtain a relatively higher accuracy comparing with single ones, and the best accuracy is achieved when three features with seven quality indexes are taken into account. For local and global features, the accuracies estimated by multiple indexes outperform single ones. This can be validated by OCL + HCS and FFT + Wavelet, respectively.

As a result, our experiments indicate that multiple features and indexes are necessary for a high accuracy quality estimation of fingerprint. Besides, different kernels (linear, polynomial and RBF) of SVM are also experimented. It can be seen that RBF kernel has the best accuracy in all indexes. 


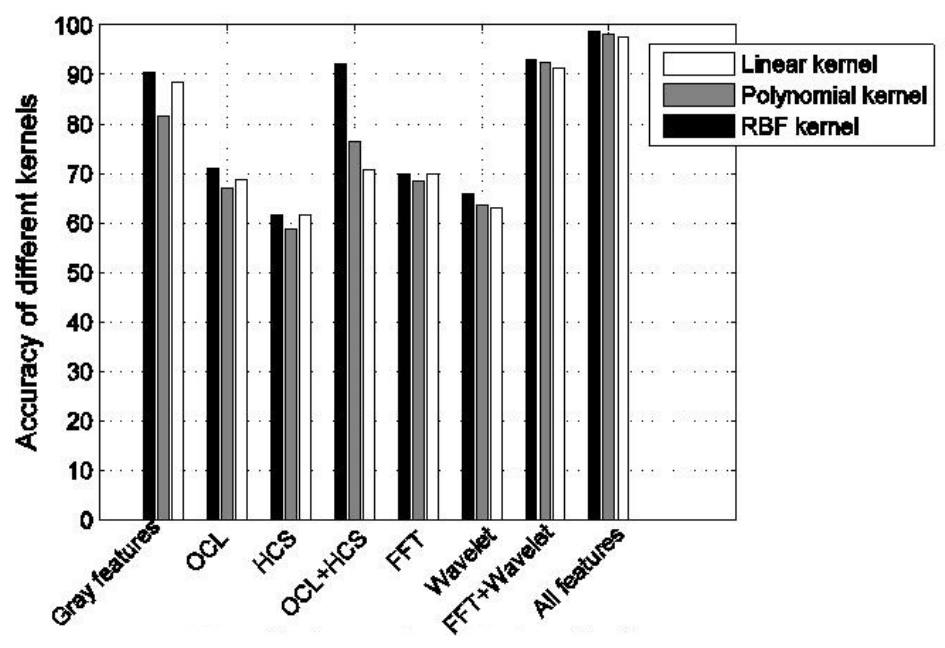

Fig.1. Accuracy comparison of different quality indexes in DB2

Accuracy and training time of different kernels under the proposed seven indexes are listed in Table 1. It can be seen the proposed estimated method (seven quality indexes based on SVM) is effective for different databases with high accuracy (more than $94 \%$ in all kernels). The RBF kernel offers the best predicting accuracy in DB1 and DB2 while achieves the worst accuracy in DB3, which implies that the RBF kernel is effective for fingerprints captured from optical sensors other than thermal devices. Training time is another metric evaluated in Table 1. DB1 has the longest training time since there are four classes in DB1. The reason of long training time in DB1 and DB2 is that a "one-against-one" approach is used, in which $k(k-1) / 2$ number of classifiers need to be constructed. RBF kernel achieves the shortest training time in DB1 and DB2 among the three kernels. It can be explained that more hyper parameters are used in polynomial than RBF kernel, and the nonlinear approach used in RBF kernel is easier to map samples to high dimension space than linear approach. As a result, RBF kernel based SVM achieves a good performance of quality estimation in optical fingerprint images.

Tab.1. Comparison of different kernels on SVM

\begin{tabular}{|c|c|c|c|c|}
\hline Performance & Dataset & Linear & Polynomial & RBF \\
\hline \multirow{3}{*}{ Accuracy } & DB1 & $95.3 \%$ & $97 \%$ & $98.5 \%$ \\
\cline { 2 - 5 } & DB2 & $97.7 \%$ & $98 \%$ & $98.7 \%$ \\
\cline { 2 - 5 } & DB3 & $97 \%$ & $94.7 \%$ & $94.3 \%$ \\
\hline \multirow{3}{*}{ Training time (s) } & DB1 & 53.981 & 1233.262 & 0.328 \\
\cline { 2 - 5 } & DB2 & 11.058 & 130.046 & 0.283 \\
\cline { 2 - 5 } & DB3 & 0.026 & 0.025 & 0.039 \\
\hline
\end{tabular}

\section{Conclusions}

A multiple indexes quality assessment for fingerprint is presented in this paper. Seven indexes from three kinds of fingerprint features are extracted and fused by a SVM classifier, which achieves a high accuracy on fingerprint quality estimation. This verified pre-process method can be melted to traditional fingerprint recognition flow to increase AFISs’ accuracy and efficiency.

\section{Acknowledgements}

This research was financially supported by the National Natural Science Foundation of China (Project Nos. 61204096 and 61404094), the Fundamental Research Fund for the Central Universities, Wuhan University (Project Nos. 2042014kf0238 and 2042015kf0174), the China Postdoctoral Science Foundation (Project No. 2012T50688), the Hubei Province Science \& Technology Pillar 
Program (2015CFB536) and the Science Foundation of Jiangsu Province, China (Project No. BK20141218).

\section{References}

[1] J. H. Chang, K. C. Fan, Fingerprint ridge allocation in direct gray-scale domain, Pattern Recognition 34 (2001) 1907-1925.

[2] E. Lim, X. Jiang, and W. Yau, Fingerprint quality and validity analysis, IEEE International Conference on Image Processing (2002) I/469-I/472.

[3] X. Tao, X. Yang, Y. Zang, X. Jia, and J. Tian, A novel measure of fingerprint image quality using Principal Component Analysis(PCA), Proceedings of the 5th IAPR International Conference on Biometrics (2012) 170-175.

[4] X. Tao, X. Yang, K. Cao, R. Wang, P. Li, and J. Tian, Estimation of fingerprint orientation field by weighted 2D fourier expansion model, Proceedings of the 20th International Conference on Pattern Recognition (2010) 1253-1256.

[5] B. Lee, J. Moon, and H. Kim, A novel measure of fingerprint image quality using Fourier spectrum, Proceedings of the International Society for Optical Engineering (2005) 105-112.

[6] N. K. Ratha, and R. Bolle, Fingerprint image quality estimation, IBM T.J. Watson Research Center, Yorktown Heights (1999).

[7] M. Wu, A. Yong, T. Zhao, and T. Guo, A systematic algorithm for fingerprint image quality assessment, 7th International Conference on Intelligent Computing (2011) 412-420.

[8] D. Yu, L. Ma, H. Lu, and Z. Chen, Fusion method of fingerprint quality evaluation: from the local gabor feature to the global spatial-frequency structures, 8th International Conference on Advanced Concepts for Intelligent Vision Systems (2006) 776-785.

[9] E. Tabassi, C. L. Wilson, and C. I. Watson, Fingerprint Image Quality, IST research report, NISTIR7151 (2004).

[10] S. J. Xie, S. Yoon, J. C. Yang, and D. S. Park, Rule-based fingerprint quality estimation system using the optimal orientation certainty level approach, Proceedings of the 2nd International Conference on Biomedical Engineering and Informatics (2009) 10.1109/BMEI.2009.5305714. 